\title{
Burckhardtsche Bestimmung der Raumgruppen II
}

\author{
Ralph Strebel
}

Ralph Strebel studierte an der ETH in Zürich Mathematik und doktorierte dort 1973 mit einer Arbeit über Gruppentheorie. Danach war er erst Assistent in Heidelberg, dann Lehrbeauftragter an verschiedenen Universitäten und Fachhochschulen. Seit 1991 ist er Professor in Freiburg im Üchtland und da insbesondere für die Ausbildung der Lehrerinnen und Lehrer der Sekundarstufe I verantwortlich.

\section{Einleitung}

Die räumliche Anordnung der Atome, Ionen oder Moleküle eines Kristalles nennt man seine Struktur; die Isometrien der Kristallstruktur bilden dann die so genannte Raumgruppe des Kristalles. Die Raumgruppen sind um 1890 von W. Barlow, von E.S. Fedorov und von A. Schoenflies mit geometrischen Methoden bestimmt worden. Gut 50 Jahre später gab Johann Jakob Burckhardt in seinem Buch Die Bewegungsgruppen der Kristallographie eine neue Herleitung dieser Gruppen. Er stützte sich dabei auf algebraische Überlegungen, die er in den dreissiger Jahren publiziert hatte ([3], [4] und [5]).

Raumgruppen sind, einfach gesagt, Symmetriegruppen der Anordnungen der Atome und Ionen eines (idealisierten) Kristalles. Mit Hilfe der Raumgruppen will man die Anordnungen der Atome und Ionen klassifizieren. Um dieses Ziel zu erreichen, muss man festlegen, wann zwei Raumgruppen als im wesentlichen gleich betrachtet werden sollen. Bei einer dieser Festsetzungen, der affinen Äquivalenz, treten 219 Klassen von Raumgruppen auf. Vertreter dieser Klassen sind bereits Ende des 19. Jahrhunderts gefunden worden. Dabei wurden in einem ersten Schritt die affinen Klassen zu noch grösseren Klassen, den Kristallklassen, zusammengefasst, und dann explizite Vertreter dieser grösseren Klassen bestimmt. Jeder dieser Vertreter legt eine Kristallklasse fest; sie ist Vereinigung von endlich vielen affinen Klassen. In einem zweiten Schritt geht es dann darum, Vertreter dieser affinen Klassen zu finden.

J.J. Burckhardt entwickelte in den dreissiger Jahren ein algebraisches Verfahren, mit dem der zweite Schritt durchgeführt werden kann. Die vorliegende und die frühere Arbeit [20] setzen sich zum Ziel, dieses Verfahren zu erklären, an Beispielen zu veranschaulichen und die Bestimmung der Raumgruppen in einen grösseren Zusammenhang einzuordnen. 
In diesem Aufsatz erkläre ich erst das Prinzip des Burckhardtschen Verfahrens und illustriere es dann durch einige Beispiele (Abschnitte 6 und 7). Das Verfahren erlaubt es, die Raumgruppen der Räume höherer Dimension zu konstruieren, falls gewisse Hilfsresultate geeignet verallgemeinert werden können. In Abschnitt 8 habe ich deshalb Ergebnisse zusammengestellt, die dies leisten und die Burckhardtschen Resultate abrunden.

Die Notation und die Definitionen, die ich im Folgenden verwende, sind jene des ersten Teils [20] dieser Arbeit: $E=(V,\langle-,-\rangle)$ bezeichnet einen Euklidischen Vektorraum und $G$ eine Raumgruppe von $E$ (Definition 3.1). Jedes Element von $G$ ist eine Isometrie von $E$, also die Komposition $\tau \circ \varphi$ einer orthogonalen Abbildung $\varphi: E \rightarrow E$ und einer Translation $\tau$ mit Vektor $\tau(0)$. Die Zuordnung $\tau \circ \varphi \mapsto(\tau(0), \varphi)$ liefert einen Isomorphismus von Iso $(E)$ auf das semi-direkte Produkt $V \rtimes \mathrm{O}(E)$. Er bildet den Normalteiler der Translationen von $G$ auf eine Untergruppe $\Gamma(G)$ von $V$ ab; diese ist ein Gitter von $E$ (Definition 2.1). Das Bild $G_{0}$ von $G$ in $O(E)$ wird Punktgruppe von $G$ genannt; $G_{0}$ ist eine Untergruppe der Symmetriegruppe $S(\Gamma(G))$ des Gitters $\Gamma$, und daher endlich (Hilfssatz 3.2).

Zwei Raumgruppen $G$ und $G^{\prime}$ werden affin äquivalent genannt, falls es eine affine Transformation $\alpha=\tau \circ L: E \rightarrow E$ gibt, so dass $G^{\prime}$ mit dem Bild der Gruppe $G$ unter der Konjugation $\tau \circ \varphi \mapsto \alpha \circ(\tau \circ \varphi) \circ \alpha^{-1}$ übereinstimmt; falls man dabei $\alpha$ orientierungserhaltend wählen kann, nennt man $G$ und $G^{\prime}$ eigentlich affin äquivalent. Sind $G$ und $G^{\prime}$ affin äquivalent, so erfüllen $\left(\Gamma=\Gamma(G), H=G_{0}\right)$ und $\left(\Gamma^{\prime}=\Gamma\left(G^{\prime}\right), H^{\prime}=G_{0}^{\prime}\right)$ die Beziehung

$$
\Gamma^{\prime}=L(\Gamma) \quad \text { und } \quad H^{\prime}=L \cdot H \cdot L^{-1} \text {; }
$$

nach Definition sind diese Paare also arithmetisch äquivalent (Definition 4.1).

\section{Prinzip des Burckhardtschen Verfahrens}

Das Burckhardtsche Verfahren geht davon aus, dass eine Liste von Paaren $(\Gamma, H)$ vorliegt, welche die arithmetischen Klassen des betrachteten Euklidischen Raumes E vertreten. Für jeden Vertreter $(\Gamma, H)$ in der Liste sucht man die affinen (oder eigentlich affinen) Äquivalenzklassen, die zur arithmetischen Klasse von $(\Gamma, H)$ gehören. Dies Ziel ist erreicht, wenn man die Familie von Raumgruppen

$$
\mathcal{M}_{(\Gamma, H)}=\left\{G \subset V \rtimes \mathrm{O}(E) \mid \Gamma(G)=\Gamma \text { und } G_{0}=H\right\}
$$

konstruieren und die Gruppen der Familie bis auf affine, oder eigentlich affine, Äquivalenz klassifizieren kann. Der Weg zum Ziel umfasst drei Etappen. Erst wird die Familie $M_{(\Gamma, H)}$ parametrisiert, und zwar durch Derivationen $D: H \rightarrow V / \Gamma$ (Nummer 6.1). Danach werden die Gruppen in $\mathcal{M}_{(\Gamma, H)}$ zu Translationsklassen zusammengefasst (Nummer 6.2). Unter der Parametrisierung entsprechen diese Klassen den Elementen einer abelschen Gruppe $H^{1}(H, V / \Gamma)$; siehe Nummer 6.3. Auf dieser Gruppe wirkt ein gewisser Normalisator $N$; seine Bahnen entsprechen unter der Parametrisierung den affinen Typen von Raumgruppen in der arithmetischen Klasse von $(\Gamma, H)$ (Nummer 6.4). Alles läuft somit darauf hinaus, die Gruppe $H^{1}(H, V / \Gamma)$ explizit berechnen und die Bahnen von $N$ bestimmen zu können. 
6.1 Parametrisierung der Familie $\mu_{(\Gamma, H)}$

Nach Definition der Punktgruppe $G_{0}=H$ gibt es für jedes $\varphi \in H$ einen Vektor $v_{\varphi} \in V$, so dass das Paar $\left(v_{\varphi}, \varphi\right)$ in $G$ liegt. Der Vektor $v_{\varphi}$ ist durch $\varphi$ nicht eindeutig bestimmt; jeder andere Vektor hat aber die Form $v_{\varphi}+u$ mit $u \in \Gamma$. Die Gruppe $G$ kann aus diesen Paaren $\left(v_{\varphi}, \varphi\right)$ zurückgewonnen werden, denn es ist

$$
G=\bigcup_{\psi \in H}\left(\Gamma+v_{\psi}, \psi\right)
$$

Nun ist $G$ aber nicht nur eine Teilmenge von Iso $(E)$, sondern eine Untergruppe; insbesondere gehört deshalb mit den Paaren $\left(v_{\varphi}, \varphi\right)$ und $\left(v_{\varphi^{\prime}}, \varphi^{\prime}\right)$ auch ihr Produkt $\left(v_{\varphi}, \varphi\right)$. $\left(v_{\varphi^{\prime}}, \varphi^{\prime}\right)=\left(v_{\varphi}+\varphi\left(v_{\varphi^{\prime}}\right), \varphi \circ \varphi^{\prime}\right)$ zu G. Dieses Produkt ist nur dann Element von $G$, wenn der Vektor $v_{\varphi}+\varphi\left(v_{\varphi^{\prime}}\right)$ in der Nebenklasse $\Gamma+v_{\varphi \circ \varphi^{\prime}}$ liegt. Folglich muss die Funktion $\psi \mapsto v_{\psi}$ die Kongruenzen

$$
v_{\varphi}+\varphi\left(v_{\varphi^{\prime}}\right) \equiv v_{\varphi \circ \varphi^{\prime}} \quad(\bmod \Gamma)
$$

für alle Paare $\left(\varphi, \varphi^{\prime}\right) \in H^{2}$ erfüllen. Diese Kongruenzen hat Frobenius bereits 1911 untersucht ([9], §5); sie werden Frobeniussche Kongruenzen genannt.

Seien umgekehrt $\psi \mapsto w_{\psi}$ eine Funktion von $H$ nach $V$, welche die Frobeniusschen Kongruenzen erfüllt, und $G^{\prime}=\bigcup_{\psi \in H}\left(\Gamma+w_{\psi}, \psi\right)$. Aus den Kongruenzen folgt dann leicht, dass $G^{\prime}$ eine Untergruppe von Iso $(E)$ ist. Weiter ist $\Gamma\left(G^{\prime}\right)=\Gamma$ und $\left(G^{\prime}\right)_{0}=H$; insbesondere ist $G^{\prime}$ eine Raumgruppe von $E$. Diese Überlegungen beweisen den

Hilfssatz 6.1 Die Vorschrift (6.2) definiert eine Parametrisierung der Familie $\mathcal{M}_{(\Gamma, H)}$ durch die Lösungen $\psi \mapsto v_{\psi}$ der Frobeniusschen Kongruenzen. Zwei Lösungen $\psi \mapsto v_{\psi}$ und $\psi \mapsto w_{\psi}$ sind dabei als gleich zu betrachten, wenn $v_{\psi}$ und $w_{\psi}$ für jedes Element $\psi \in H$ kongruent modulo $\Gamma$ sind.

6.2 Übergang zu Translationsklassen in $\boldsymbol{M}_{(\Gamma, H)}$

Hilfssatz 6.1 parametrisiert die Familie $\mathcal{M}_{(\Gamma, H)}$; wir interessieren uns aber nicht so sehr für sie, als für die Menge der affinen Klassen von Raumgruppen, die $M_{(\Gamma, H)}$ schneiden. Wie Burckhardt erkannte, findet man diese Klassen am besten in zwei Schritten. Im ersten konjugiert man die Raumgruppen $G$ nur mit Translationen; dies führt auf eine Äquivalenzrelation, welche die affine Äquivalenz verfeinert und Translationsäquivalenz genannt wird. Danach untersucht man dann die Auswirkung des Konjugierens der Raumgruppen in $\mathcal{M}_{(\Gamma, H)}$ unter linearen Transformationen. Die Rechnung

$$
(u, \mathbb{1}) \cdot(v, \varphi) \cdot(u, \mathbb{1})^{-1}=(u+v, \varphi) \cdot(-u, \mathbb{1})=(v+(\mathbb{1}-\varphi)(u), \varphi)
$$

lehrt zunächst, dass Konjugation mit der Translation $\tau_{u}: x \mapsto x+u$ die Menge $\mathcal{M}_{(\Gamma, H)}$ in sich abbildet, und dass die Gruppe $G \in \mathcal{M}_{(\Gamma, H)}$ mit der Parametrisierung $\psi \mapsto v_{\psi}$ dabei in die Gruppe $G^{\prime}$ mit der Parametrisierung

$$
\psi \mapsto v_{\psi}^{\prime}=v_{\psi}+(\mathbb{1}-\psi)(u)
$$


überführt wird. Frobenius folgerte aus dieser Transformationsformel, dass $\mathcal{M}_{(\Gamma, H)}$ aus endlich vielen Translationsklassen zusammengesetzt ist; er ging so vor: Die Parametrisierung $\psi \mapsto v_{\psi}$ erfüllt die Kongruenzen (6.3); summiert man diese für festes $\varphi$ über alle $\varphi^{\prime} \in H$, bekommt man die Beziehung

$$
|H| \cdot v_{\varphi}+\varphi\left(\sum_{\varphi^{\prime} \in H} v_{\varphi^{\prime}}\right) \equiv \sum_{\varphi^{\prime} \in H} v_{\varphi \circ \varphi^{\prime}} \quad(\bmod \Gamma)
$$

Nun durchläuft mit $\varphi^{\prime}$ auch $\varphi \circ \varphi^{\prime}$ alle Elemente von $H$. Setzt man also $h=\operatorname{card}(H)$ und $w=\frac{1}{h} \sum_{\varphi^{\prime} \in H} v_{\varphi^{\prime}}$, so folgt aus obiger Summierung die Kongruenz

$$
h \cdot v_{\varphi} \equiv(\mathbb{1}-\varphi)(h \cdot w) \quad(\bmod \Gamma)
$$

für jedes Element $\varphi \in H$. Die Parametrisierung $\varphi \mapsto v_{\varphi}^{\prime}=v_{\varphi}-(\mathbb{1}-\varphi)(w)$ gehört zur Raumgruppe $G^{\prime}=\tau_{-w} \cdot G \cdot \tau_{-w}^{-1}$; da die Vektoren $v_{\varphi}^{\prime}$ die Kongruenzen $h \cdot v_{\varphi}^{\prime} \equiv 0$ modulo $\Gamma$ erfüllen, liegen sie in $\frac{1}{h} \Gamma$. Sei nun $\mathscr{B}=\left(b_{1}, \ldots, b_{n}\right)$ eine $\mathbb{Z}$-Basis von $\Gamma$. Jeder Vektor $v_{\varphi}^{\prime}$ ist dann eine Linearkombination der Form $\sum_{1 \leq \ell \leq n} m_{\ell} / h \cdot b_{\ell}$ mit ganzzahligen Koeffizienten $m_{\ell}$. Weil die Parametrisierung von $G^{\prime}$ nur bis auf Summanden aus $\Gamma$ bestimmt ist, können wir annehmen, jede der Zahlen $m_{\ell}$ liege in der endlichen Menge $\{0,1,2, \ldots, h-1\}$. Deshalb gilt der

Satz 6.2 Seien $\Gamma$ ein Gitter in $E^{n}$ und $H$ eine Untergruppe von $\mathrm{S}(\Gamma)$ mit $h$ Elementen. Dann besteht $\mathcal{M}_{(\Gamma, H)}$ aus höchstens $\left(h^{n}\right)^{h}$ Translationsklassen.

6.3 Einführung der Kohomologiegruppe $H^{1}(H, V / \Gamma)$

J.J. Burckhardt gelang es, die vorgeführte Rechnung von Frobenius so zu verfeinern, dass er die Translationsklassen in $\mathcal{M}_{(\Gamma, H)}$ explizit bestimmen konnte. In der Einleitung der Arbeit [4] äussert er sich zu diesem Vorhaben wie folgt:

Ich werde aus den Ansätzen von Frobenius heraus gewisse Sätze herleiten, die für zyklische Gruppen und Gruppen, die sich aus solchen zyklischen Gruppen zusammensetzen, diese Verzweigung der [arithmetischen] Klassen in die verschiedenen Bewegungsgruppen regeln. Dadurch ist dann zugleich die Aufgabe gelöst, die Bieberbach-Frobenius'sche Methode soweit durchzuführen, daß man zu gegebener Klasse die zugehörigen Gruppen angeben kann.

Im Folgenden skizziere ich die Burckhardtschen Überlegungen und Resultate; ich werde mich aber nicht an seine Darstellung halten, sondern Begriffe, Bezeichnungen und Methoden der heutigen Algebra verwenden.

Jede Gruppe $G \in \mathcal{M}_{(\Gamma, H)}$ gibt Anlass zu Funktionen $\psi \mapsto v_{\psi}$, deren Werte in $V$ liegen, aber nur modulo $\Gamma$ eindeutig bestimmt sind. Setzt man diese Funktionen mit der kanonischen Projektion $V \rightarrow V / \Gamma$ zusammen, erhält man die Funktion

$$
D_{G}: H \rightarrow V / \Gamma, \quad \psi \mapsto v_{\psi}+\Gamma
$$

sie erfüllt die Identitäten

$$
D_{G}\left(\varphi \circ \varphi^{\prime}\right)=D_{G}(\varphi)+\bar{\varphi}\left(D_{G}\left(\varphi^{\prime}\right)\right) \text {. }
$$


In ihnen bezeichnet $\bar{\varphi}$ den von $\varphi$ auf $V / \Gamma$ induzierten Automorphismus von Gruppen. Er existiert, da $\varphi$ das Gitter $\Gamma$ auf sich abbildet; er ordnet der Klasse $v+\Gamma$ die Klasse $\varphi(v)+\Gamma$ zu. Jede Funktion $D: H \rightarrow V / \Gamma$, welche die Identitäten (6.8) erfüllt, wird Derivation oder verschränkter Homomorphismus genannt. Die Menge der Derivationen von $H$ mit Werten in $V / \Gamma$ bezeichne ich mit $\operatorname{Der}(H, V / \Gamma)$.

Hilfssatz 6.1 besagt, dass die Vorschrift $G \mapsto D_{G}$ eine Bijektion von $\mathcal{M}_{(\Gamma, H)}$ auf $\operatorname{Der}(H, V / \Gamma)$ liefert. Nun sind die Elemente von $\operatorname{Der}(H, V / \Gamma)$ aber Funktionen, die auf die übliche Art addiert werden können. Wie man leicht nachprüft, ist $\operatorname{Der}(H, V / \Gamma)$ eine Untergruppe der Gruppe aller Funktionen $f: H \rightarrow V / \Gamma$.

Die Konstruktion von Derivationen ist im Allgemeinen mühsam; einige sind aber leicht zu finden. Jedes Element $a \in V / \Gamma$ gibt nämlich Anlass zu einer so genannten inneren Derivation $D_{a}$, definiert durch $\psi \mapsto(\mathbb{1}-\bar{\psi})(a)$; die Rechnung

$$
D_{a}\left(\varphi \circ \varphi^{\prime}\right)=\left(\mathbb{1}-\bar{\varphi} \circ \overline{\varphi^{\prime}}\right)(a)=\left(\mathbb{1}-\bar{\varphi}+\bar{\varphi} \circ\left(\mathbb{1}-\overline{\varphi^{\prime}}\right)\right)(a)=D_{a}(\varphi)+\bar{\varphi}\left(D_{a}\left(\varphi^{\prime}\right)\right)
$$

zeigt, dass $D_{a}$ auch wirklich eine Derivation ist. Die inneren Derivationen von $H$ mit Werten in $V / \Gamma$ bilden eine Untergruppe von $\operatorname{Der}(H, V / \Gamma)$; ich bezeichne sie mit $\operatorname{Inn}(H, V / \Gamma)$. Die Formel (6.5) lehrt dann, dass die Translationsklassen der Raumgruppen in $M_{(\Gamma, H)}$ durch die Korrespondenz $G \mapsto D_{G}$ auf die Nebenklassen von $\operatorname{Inn}(H, V / \Gamma)$ in $\operatorname{Der}(H, V / \Gamma)$ abgebildet werden. Führt man die Faktorgruppe

$$
H^{1}(H, V / \Gamma)=\operatorname{Der}(H, V / \Gamma) / \operatorname{Inn}(H, V / \Gamma)
$$

ein, lässt sich diese Erkenntnis so aussprechen:

Satz 6.3 Die Korrespondenz $G \mapsto D_{G}$ induziert eine Bijektion $\eta_{(\Gamma, H)}$ zwischen der Menge der Translationsklassen in $\mathcal{M}_{(\Gamma, H)}$ und der Gruppe $H^{1}(H, V / \Gamma)$.

Die Gruppe $H^{1}(H, V / \Gamma)$ wird erste Kohomologiegruppe von $H$ mit Koeffizienten in $V / \Gamma$ genannt. Sie ist endlich, nach Satz 6.2 ist ihre Ordnung nämlich höchstens $h^{h n}$ (es bezeichnet $n$ die Dimension von $V$ ).

6.4 Wirkung des Normalisators auf der Gruppe $H^{1}(H, V / \Gamma)$

In dieser Nummer bestimme ich die Form der Teilmengen von $H^{1}(H, V / \Gamma)$, die unter $\eta_{(\Gamma, H)}$ den Teilmengen von affin äquivalenten Translationsklassen entsprechen.

Seien $G \in \mathcal{M}_{(\Gamma, H)}$ und $[G]_{\text {aff }}$ die affine Äquivalenzklasse von $G$. Da $[G]_{\text {aff }}$ eine Vereinigung von Translationsklassen ist, besteht der Durchschnitt $[G]_{\text {aff }} \cap \mathcal{M}_{(\Gamma, H)}$ aus vollen Translationsklassen; unter der Korrespondenz $\eta_{(\Gamma, H)}$ entspricht ihm eine Teilmenge $B_{G}$ von $H^{1}(H, V / \Gamma)$. Um ihre Form zu finden, betrachte ich eine lineare Transformation $L: V \rightarrow V$ und ein Element $(v, \varphi)$ von $G$. Die Rechnung

$$
(0, L) \cdot(v, \varphi) \cdot(0, L)^{-1}=(L(v), L \circ \varphi) \cdot\left(0, L^{-1}\right)=\left(L(v), L \circ \varphi \circ L^{-1}\right)
$$

zeigt dann, dass die konjugierte Raumgruppe $G^{\prime}=L \cdot G \cdot L^{-1}$ genau dann in $M_{(\Gamma, H)}$ liegt, falls $L$ das Gitter $\Gamma$ auf sich abbildet und $L \cdot H \cdot L^{-1}$ mit $H$ übereinstimmt. Es 
gehört $L$ also zum Normalisator $N=N_{\mathrm{GL}(\Gamma)}(H)$ von $H$ in der Gruppe all jener linearen Transformationen $L$ von $V$, die das Gitter $\Gamma$ invariant lassen.

Seien nun $\psi \mapsto v_{\psi}$ eine Funktion, die $G$ parametrisiert, und $L \in N$. Aus obiger Rechnung folgt, dass die konjugierte Gruppe $G^{\prime}=L \cdot G \cdot L^{-1}$ durch die Funktion $L \circ \psi \circ L^{-1} \mapsto L\left(v_{\psi}\right)$ parametrisiert wird; ihre Standardbeschreibung ist $\psi \mapsto L\left(v_{L^{-1} \circ \psi_{\circ} L}\right)$. Somit bildet die lineare Transformation $L$ die Derivation $D_{G}$ auf

$$
D_{G^{\prime}}=L_{*}\left(D_{G}\right): \psi \longmapsto \bar{L}\left(D\left(L^{-1} \circ \psi \circ L\right)\right)
$$

ab. Die Menge $B_{G}$ ist also eine Bahn der Wirkung von $N$ auf $H^{1}(H, V / \Gamma)$. Verwendet man in obiger Überlegung die eigentlich affine Äquivalenz von Raumgruppen, hat man den Normalisator $N$ durch $N^{+}=N_{\mathrm{SL}(\Gamma)}(H)$ zu ersetzen. Insgesamt erhält man daher

Theorem 6.4 Die Menge der affinen (oder eigentlich affinen) Äquivalenzklassen von Raumgruppen, die zur arithmetischen Klasse $[(\Gamma, H)]$ gehören, entspricht bijektiv den Bahnen des Normalisators $N_{\mathrm{GL}(\Gamma)}(H)$ (oder $\left.N_{\mathrm{SL}(\Gamma)}(H)\right)$ auf der Gruppe $H^{1}(H, V / \Gamma)$.

\subsection{Konstruktion von Derivationen}

Im folgenden Hilfssatz werden einige Eigenschaften der Derivationen zusammengestellt, die sich durch einfache Rechnungen begründen lassen. Da später Derivationen mit Werten in verschiedenen Moduln benützt werden, gehe ich vom $H$-Modul $V / \Gamma$ zu einem beliebigen $H$-Modul $M$ über; die Beweise werden dadurch nicht schwieriger, die Notation sogar einfacher. (Ein $H$-Modul ist eine abelsche Gruppe, für welche ein Homomorphismus $H \rightarrow \operatorname{Aut}(M)$ festgelegt worden ist.)

Hilfssatz 6.5 Seien $H$ eine Gruppe, $M$ ein H-Modul und $D: H \rightarrow M$ eine Derivation. Dann gelten die Aussagen:

(i) $D(\mathbb{1})=0$,

(ii) $D\left(\varphi^{-1}\right)=-\varphi^{-1}(D(\varphi))$ für $\varphi \in H$.

(iii) $D\left(\varphi^{k}\right)=\left(\mathbb{1}+\varphi+\cdots+\varphi^{k-1}\right)(D(\varphi))$ für $\varphi \in H$ und $k>1$.

(iv) Erzeugt $\mathscr{Y}$ die Gruppe $H$, so wird $D$ bereits durch die Werte auf $\mathscr{Y}$ festgelegt.

Will man die Gruppe $H^{1}(H, V / \Gamma)=\operatorname{Der}(H, V / \Gamma) / \operatorname{Inn}(H, V / \Gamma)$ explizit bestimmen, muss man Derivationen konstruieren können. Der nächste Hilfssatz zeigt, wie diese Aufgabe für zyklische Gruppen gelöst werden kann.

Hilfssatz 6.6 Seien $H$ eine zyklische Gruppe der Ordnung $h>1$ und $M$ ein $H$ Modul. Ist $\varphi_{1}$ ein Element, das $H$ erzeugt, so liefert die Auswertung $D \mapsto D\left(\varphi_{1}\right)$ einen Isomorphismus von $\operatorname{Der}(H, M)$ auf den Kern $\operatorname{ker} \delta$ des Endomorphismus

$$
\delta=\mathbb{1}+\varphi_{1}+\varphi_{1}^{2}+\cdots+\varphi_{1}^{h-1} .
$$


Beweis. Ist $D: H \rightarrow M$ eine Derivation, so gilt nach Hilfssatz 6.5 die Rechnung

$$
0=D(\mathbb{1})=D\left(\varphi_{1}^{h}\right)=\left(\mathbb{1}+\varphi_{1}+\cdots+\varphi_{1}^{h-1}\right)\left(D\left(\varphi_{1}\right)\right)=\delta\left(D\left(\varphi_{1}\right)\right) .
$$

Sie zeigt, dass $D\left(\varphi_{1}\right)$ im Kern von $\delta$ liegt. Sei umgekehrt $a \in \operatorname{ker} \delta$. Die Vorschrift $\varphi_{1}^{k} \longmapsto\left(\mathbb{1}+\varphi_{1}+\varphi_{1}^{2}+\cdots+\varphi_{1}^{k-1}\right)(a)$ definiert eine Funktion von $H$ mit Werten in $M$; eine kurze Rechnung zeigt, dass es sich bei ihr um eine Derivation $D_{1}$ handelt; nach Punkt (iii) von Hilfssatz 6.5 ist sie die einzige Derivation mit $D_{1}\left(\varphi_{1}\right)=a$.

Derivationen von komplizierteren Gruppen lassen sich aus Hilfssatz 6.6 mit Hilfe des nächsten Resultates gewinnen. In ihm geht es um eine Gruppe $H$ mit zwei Untergruppen $S$ und $T$, in der jedes Element $\psi \in H$ eindeutig in den Formen $s \cdot t$ und $\bar{t} \cdot \bar{s}$ mit $s, \bar{s}$ in $S$ sowie $t, \bar{t}$ in $T$ geschrieben werden kann.

Hilfssatz 6.7 Seien $S$ und $T$ Untergruppen der Punktgruppe $H$ mit $S \cap T=\{\mathbb{1}\}$ und $H=S \cdot T$. Seien weiter $M$ ein $H$-Modul und $D_{S}: S \rightarrow M$ sowie $D_{T}: T \rightarrow M$ Derivationen. Dann ist die Funktion

$$
f: H \rightarrow M, \quad \psi=s \cdot t \mapsto D_{S}(s)+s \cdot D_{T}(t)
$$

genau dann eine Derivation von H, falls die Gleichungen

$$
D_{S}(s)+s \cdot D_{T}(t)=D_{T}(\bar{t})+\bar{t} \cdot D_{S}(\bar{s})
$$

für alle Paare $(s, t) \in S \times T$ erfüllt sind.

Beweis. Falls $f$ eine Derivation ist, muss ihr Wert auf $\psi=s \cdot t=\bar{t} \cdot \bar{s}$ unabhängig von der Darstellung von $\psi$ sein, weshalb die Gleichungen (6.11) erfüllt sein müssen. Nehmen wir nun an, sie treffen zu. Seien $\psi=s \cdot \bar{t}, \psi^{\prime}=\bar{s}^{\prime} \cdot t^{\prime}$ und seien $s^{\prime} \in S^{\prime}$ sowie $t \in T$ so gewählt, dass die Beziehung $\bar{t} \cdot \bar{s}^{\prime}=s^{\prime} \cdot t$ gilt. Die folgende Rechnung zeigt, dass $f$ eine Derivation ist.

$$
\begin{aligned}
f\left(\psi \cdot \psi^{\prime}\right) & =f\left(s \bar{t} \cdot \bar{s}^{\prime} t^{\prime}\right)=f\left(s \cdot s^{\prime} t \cdot t^{\prime}\right) \\
& =\left(D_{S}(s)+s \cdot D_{S}\left(s^{\prime}\right)\right)+s s^{\prime} \cdot\left(D_{T}(t)+t \cdot D_{T}\left(t^{\prime}\right)\right) \\
& =D_{S}(s)+s \cdot\left(D_{S}\left(s^{\prime}\right)+s^{\prime} \cdot D_{T}(t)\right)+s \cdot s^{\prime} t \cdot D_{T}\left(t^{\prime}\right) \\
& =D_{S}(s)+s \cdot\left(D_{T}(\bar{t})+\bar{t} \cdot D_{S}\left(\bar{s}^{\prime}\right)\right)+s \cdot \bar{s}^{\prime} \cdot D_{T}\left(t^{\prime}\right) \\
& =f(\psi)+\psi \cdot f\left(\psi^{\prime}\right) .
\end{aligned}
$$

\section{Bemerkungen.}

1) In [4] beweist Burckhardt einen Spezialfall von Hilfssatz 6.7 (S. 176-177; s. [6], S. 118, Satz 35). Damit berechnet er dann schrittweise die Kohomologiegruppen aller kristallographischen Gruppen des 3-dimensionalen Raumes.

2) Die schrittweise Konstruktion von Derivationen ähnelt der Schoenfliesschen Untersuchungsmethode im Falle nicht zyklischer Punktgruppen (siehe [20], Nummer 4.2, Bemerkung 2). 
6.6 Berechnung der Gruppe $H^{1}(H, V / \Gamma)$

Die Elemente der Gruppe $H^{1}(H, V / \Gamma)$ sind die Nebenklassen der Untergruppe $\operatorname{Inn}(H, V / \Gamma)$ in $\operatorname{Der}(H, V / \Gamma)$. Burckhardt findet sie, indem er eine gegebene Derivation $D: H \rightarrow V / \Gamma$ mit Hilfe von inneren Derivationen in eine Derivation $D^{\prime}$ besonders einfacher Gestalt überführt; $D^{\prime}$ wird dabei durch gewisse Parameterwerte beschrieben. Falls $D^{\prime}$ die Nullfunktion ist, steht fest, dass jede Derivation eine innere ist, die Kohomologiegruppe also nur aus einem Element besteht. Dieser Fall liegt zum Beispiel vor, wenn $H$ zyklisch und die Zahl 1 nicht Eigenwert der erzeugenden Abbildung $\varphi_{1}: V \rightarrow V$ ist ([4], Sätze 1 und 3). Falls $D^{\prime}$ nicht die Nullfunktion ist, weist Burckhardt nach, dass die Funktionen $D^{\prime}$ für alle noch zugelassenen Werte der Parameter tatsächlich Derivationen sind und dass verschiedene Parameterwerte zu nicht äquivalenten Derivationen führen.

Im Folgenden schlage ich einen anderen Weg ein. Auf ihm werden zwei endlich erzeugbare Hilfsgruppen von Derivationen $\operatorname{Der}\left(H, \Gamma_{h}\right)$ und $\operatorname{Der}\left(H, \Gamma_{h} / \Gamma\right)$ berechnet und $H^{1}(H, V / \Gamma)$ als Kokern einer Abbildung $\pi_{*}: \operatorname{Der}\left(H, \Gamma_{h}\right) \rightarrow \operatorname{Der}\left(H, \Gamma_{h} / \Gamma\right)$ gewonnen. Ausgangspunkt des Weges ist die Frobeniussche Überlegung, die zu Satz 6.2 führte. Gemäss ihr induziert die Inklusion $\mu: \frac{1}{h} \Gamma / \Gamma \hookrightarrow V / \Gamma$ einen surjektiven Homomorphismus

$$
\mu_{*}: \operatorname{Der}\left(H, \frac{1}{h} \Gamma / \Gamma\right) \rightarrow H^{1}(H, V / \Gamma)=\operatorname{Der}(H, V / \Gamma) / \operatorname{Inn}(H, V / \Gamma) .
$$

Bezeichnet man den Teilmodul $\frac{1}{h} \Gamma=\left\{\frac{1}{h} w \mid w \in \Gamma\right\}$ von $V$ mit $\Gamma_{h}$, kann der Kern von $\mu_{*}$ wie folgt beschrieben werden:

Satz 6.8 Sei $\pi$ die kanonische Projektion von $\Gamma_{h}$ auf $\Gamma_{h} / \Gamma$. Dann ist

$$
\operatorname{ker} \mu_{*}=\operatorname{im}\left(\pi_{*}: \operatorname{Der}\left(H, \Gamma_{h}\right) \rightarrow \operatorname{Der}\left(H, \Gamma_{h} / \Gamma\right)\right) .
$$

Beweis. Ist $D \in \operatorname{ker} \mu_{*}$, so liegt $\mu \circ D$ in $\operatorname{Inn}(H, V / \Gamma)$; nach Definition gibt es also ein Element $a=v+\Gamma \in V / \Gamma$, so dass die Gleichung $D(\psi)=(\mathbb{1}-\psi)(v)+\Gamma$ für alle $\psi \in H$ erfüllt ist. Da $D$ eine Derivation mit Werten in $\Gamma_{h} / \Gamma$ ist, sind die Vektoren $(\mathbb{1}-\psi)(v)$ Elemente von $\Gamma_{h}$; die Zuordnung $\psi \mapsto(\mathbb{1}-\psi)(v)$ ist daher eine Derivation $\tilde{D} \in \operatorname{Der}\left(H, \Gamma_{h}\right)$ mit $\pi \circ \tilde{D}=D$.

Ist umgekehrt $\tilde{D} \in \operatorname{Der}\left(H, \Gamma_{h}\right)$, so setze man $w=\frac{1}{h} \sum_{\psi \in H} \tilde{D}(\psi)$. Wie in Nummer 6.2, Gleichung (6.6), ergibt sich dann die Beziehung $h \cdot \tilde{D}(\varphi)=(\mathbb{1}-\varphi)(h \cdot w)$. Da die Division durch $h$ in $V$ definiert ist, impliziert sie die Gleichung $\tilde{D}(\varphi)=(\mathbb{1}-\varphi)(w)$ für jedes $\varphi \in H$; diese zeigt, dass $\mu_{*}\left(\pi_{*}(\tilde{D})\right)=\mu \circ \pi \circ \tilde{D}$ zu $\operatorname{Inn}(H, V / \Gamma)$ gehört.

\section{Beispiele des Burckhardtschen Verfahrens}

In diesem Abschnitt zeige ich an 3 Beispielen auf, wie Vertreter der eigentlich affinen Typen der Raumgruppen mit dem Burckhardtschen Verfahren gewonnen werden können. Ich beginne mit der geometrischen Klasse einer zyklischen Drehgruppe der Ordnung 4; diese geometrische Klasse besteht aus den arithmetischen Klassen P4 und I4 (siehe [20], Beispiel in 4.2). 


\subsection{Affine Typen von Raumgruppen in der Klasse $P 4$}

Seien $\rho: \mathbb{R}^{3} \rightarrow \mathbb{R}^{3}$ die Drehung $\left(x_{1}, x_{2}, x_{3}\right)^{t} \mapsto\left(-x_{2}, x_{1}, x_{3}\right)^{t}$ und $H$ die von $\rho$ erzeugte Gruppe. Das Paar $\left(\mathbb{Z}^{3}, H\right)$ liegt in der Klasse P4. Um die Kohomologiegruppe $H^{1}\left(H, \mathbb{R}^{3} / \mathbb{Z}^{3}\right)$ mit Satz 6.8 zu bestimmen, berechnen wir mit Hilfssatz 6.6 die Gruppen $\operatorname{Der}(H, M)$ und $\operatorname{Der}(H, \bar{M})$ für die beiden Module $M=\left(\frac{1}{4} \mathbb{Z}\right)^{3}$ und $\bar{M}=\left(\frac{1}{4} \mathbb{Z} / \mathbb{Z}\right)^{3}$. Der Endomorphismus $\delta=\mathbb{1}+\rho+\rho^{2}+\rho^{3}$ von $\mathbb{R}^{3}$ ergibt sich zu

$$
\delta\left(\left(x_{1}, x_{2}, x_{3}\right)^{t}\right)=\left(0,0,4 x_{3}\right)^{t} .
$$

Der Kern der Einschränkung von $\delta$ auf $M$ ist gleich $\frac{1}{4} \mathbb{Z} \oplus \frac{1}{4} \mathbb{Z} \oplus\{0\}$; dessen Bild in $\operatorname{ker} \bar{\delta}$ ist $\bar{M}_{1}=\left(\frac{1}{4} \mathbb{Z} / \mathbb{Z}\right) \oplus\left(\frac{1}{4} \mathbb{Z} / \mathbb{Z}\right) \oplus\{0\}$. Auf $\bar{M}$ induziert $\delta$ die Nullabbildung; also ist $\operatorname{ker} \bar{\delta}=\bar{M}$. Die Quotientengruppe $\bar{M} / \bar{M}_{1}$ ist deshalb isomorph mit $\frac{1}{4} \mathbb{Z} / \mathbb{Z}$. Es folgt, dass die Gruppe $H^{1}\left(H, \mathbb{R}^{3} / \mathbb{Z}^{3}\right)$ die Ordnung 4 hat und durch die Nebenklasse der Derivation

$$
D: H \rightarrow \mathbb{R}^{3} / \mathbb{Z}^{3}, \quad D\left(\rho^{k}\right)=k \cdot u+\mathbb{Z}^{3} \text { mit } u=\left(0,0, \frac{1}{4}\right)^{t} \text { und } k=0,1,2,3
$$

erzeugt wird. Die Menge $\mathcal{M}_{\left(\mathbb{Z}^{3}, H\right)}$ besteht aus 4 Translationsklassen (Satz 6.3); sie werden durch die 4 Gruppen

$$
G_{\ell}=\left(\mathbb{Z}^{3}, \mathbb{1}\right) \cup\left(\mathbb{Z}^{3}+\ell \cdot u, \rho\right) \cup\left(\mathbb{Z}^{3}+2 \ell \cdot u, \rho^{2}\right) \cup\left(\mathbb{Z}^{3}+3 \ell \cdot u, \rho^{3}\right)
$$

vertreten. Die Gruppe $G_{0}$ ist das semi-direkte Produkt $\mathbb{Z}^{3} \rtimes H$; sie enthält Drehungen der Ordnung 4. Die Gruppe $G_{1}$ wird erzeugt durch die Translationen mit Translationsvektoren aus $\mathbb{Z}^{3}$ und durch die Schraubung $x \mapsto \rho(x)+(0,0,1 / 4)^{t}$ um die $x_{3}$-Achse; die Isometrien in $G_{1}$ sind entweder Translationen oder Schraubungen; sie haben also alle unendliche Ordnung (abgesehen von $\mathbb{1}$ ). Die Gruppe $G_{2}$ hat keine Drehung der Ordnung 4 , wohl aber eine Drehung der Ordnung 2 , nämlich $\left((0,0,-1)^{t}+2 \cdot 2 \cdot u, \rho^{2}\right)$. Die Gruppe $G_{3}$ enthält, genau wie $G_{1}$, nur Translationen und Schraubungen.

Die genannten Eigenschaften der Gruppen $G_{0}, G_{1}$ und $G_{2}$ garantieren, dass sie paarweise nicht isomorph, also insbesondere nicht affin äquivalent, sind. Dieses Ergebnis wird durch Theorem 6.4 bestätigt und ergänzt. Nach ihm bildet $\eta_{\left(\mathbb{Z}^{3}, H\right)}$ die nicht-leeren Durchschnitte $M_{\left(\mathbb{Z}^{3}, H\right)} \cap[G]_{\text {aff }}$ bijektiv auf die Bahnen ab, welche der Normalisator $N=N_{\mathrm{GL}\left(\mathbb{Z}^{3}\right)}(H)$ auf $H^{1}\left(H, \mathbb{R}^{3} / \mathbb{Z}^{3}\right)$ erzeugt. Da die Kohomologiegruppe zyklisch von der Ordnung 4 ist, lässt sie nur die Automorphismen $\mathbb{1}_{*}$ und $-\mathbb{1}_{*} \mathrm{zu}$; beide halten das neutrale Element $\operatorname{Inn}\left(H, \mathbb{R}^{3} / \mathbb{Z}^{3}\right)$ und das Element $2 D+\operatorname{Inn}\left(H, \mathbb{R}^{3} / \mathbb{Z}^{3}\right)$ fest; hingegen vertauscht $-\mathbb{1}_{*}$ die beiden anderen Elemente. Die Gruppen $G_{1}$ und $G_{3}$ sind also genau dann affin äquivalent, beziehungsweise eigentlich affin äquivalent, wenn der Automorphismus $-\mathbb{1}_{*}$ auf $H^{1}\left(H, \mathbb{R}^{3} / \mathbb{Z}^{3}\right)$ durch ein Element von $N$, beziehungsweise von $N^{+}$, induziert wird.

Die Inversion $-1: \mathbb{Z}^{3} \rightarrow \mathbb{Z}^{3}$ gehört zu $N$ und transformiert jede Derivation in ihr Negatives, denn nach Gleichung (6.10) ist

$$
(-\mathbb{1})_{*}(D): \psi \longmapsto \overline{(-\mathbb{1})}(D(-\mathbb{1} \circ \psi \circ-\mathbb{1}))=-D(\psi) .
$$

Die Raumgruppen $G_{1}$ und $G_{3}$ sind also affin äquivalent. Sie sind aber nicht eigentlich affin äquivalent. Um dies zu begründen, muss der Normalisator $N^{+}=N_{\mathrm{SL}\left(\mathbb{Z}^{3}\right)}(H)$ bestimmt 
werden. Er umfasst die Diedergruppe, die von $\rho$ und der Drehung $\rho^{\prime}: x \mapsto\left(x_{1},-x_{2},-x_{3}\right)^{t}$ erzeugt wird; eine Hilfsrechnung zeigt weiter, dass $N^{+}$mit dieser Diedergruppe zusammenfällt. Transformiert man $D$ mit $\rho$ und $\rho^{\prime}$, wird $D$ festgehalten, denn es sind

$$
\left(\rho_{*}(D)\right)(\rho)=\rho(D(\rho))=\rho(u)=u \text { und }\left(\rho_{*}^{\prime}(D)\right)(\rho)=\rho^{\prime}\left(D\left(\rho^{-1}\right)\right)=\rho^{\prime}(-u)=u \text {. }
$$

Nach Theorem 6.4 gibt es daher 4 eigentlich affine Typen von Raumgruppen in der arithmetischen Klasse P4; die Kristallographen bezeichnen sie mit $P 4, P 4_{1}, P 4_{2}$ und $P 4_{3}$.

Bemerkung. Ein Vergleich der algebraischen Methode mit jener von Schoenflies ist lehrreich. Schoenflies gewinnt die Typen der Raumgruppen, indem er erst die möglichen Ganghöhen der Schraubungen bestimmt; dies entspricht der Berechnung der Gruppe $\operatorname{Der}\left(H,\left(\frac{1}{4} \mathbb{Z} / \mathbb{Z}\right)^{3}\right)$. Danach analysiert er die Anordnung der Achsen und erkennt, gestützt auf die räumliche Anschauung, dass es 4 verschiedene Typen von Raumgruppen gibt. Beim algebraischen Zugang fällt die Hilfe der Anschauung weg; sie wird durch den Existenznachweis von Derivationen und die Bestimmung der Bahnen auf $H^{1}\left(H, \mathbb{R}^{3} / \mathbb{Z}^{3}\right)$ ersetzt.

\subsection{Affine Typen von Raumgruppen in der Klasse I4}

Das innenzentrierte Gitter $\Gamma=\mathbb{Z}^{3} \cup \mathbb{Z}^{3}+\left(\frac{1}{2}, \frac{1}{2}, \frac{1}{2}\right)^{t}$ hat die Basis $b_{1}=e_{1}, b_{2}=e_{2}$ und $b_{3}=\left(\frac{1}{2}, \frac{1}{2}, \frac{1}{2}\right)^{t}$. In Koordinaten bezüglich dieser Basis $\mathscr{B}$ hat $\rho$ die Beschreibung

$$
\rho_{\mathscr{B}}:\left(y_{1}, y_{2}, y_{3}\right)^{t} \longmapsto\left(-y_{2}-y_{3}, y_{1}, y_{3}\right)^{t}
$$

und der Endomorphismus $\delta$ wird zu $\delta_{\mathscr{B}_{3}}:\left(y_{1}, y_{2}, y_{3}\right)^{t} \mapsto\left(-2 y_{3},-2 y_{3}, 4 y_{3}\right)^{t}$. Die Kerne von $\left(\delta_{\mathscr{B}}\right)$ und $\overline{\delta_{\mathscr{B}}}$ auf $M=\left(\frac{1}{4} \mathbb{Z}\right)^{3}$ und $\bar{M}=\left(\frac{1}{4} \mathbb{Z} / \mathbb{Z}\right)^{3}$ sind

$$
\operatorname{ker}\left(\delta_{\mathscr{B}}\right)_{\mid M}=\frac{1}{4} \mathbb{Z} \oplus \frac{1}{4} \mathbb{Z} \oplus\{0\} \quad \text { und } \operatorname{ker} \overline{\delta_{\mathscr{B}}}=\frac{1}{4} \mathbb{Z} / \mathbb{Z} \oplus \frac{1}{4} \mathbb{Z} / \mathbb{Z} \oplus \frac{1}{2} \mathbb{Z} / \mathbb{Z}
$$

Die Gruppe $H^{1}\left(H, \mathbb{R}^{3} / \Gamma\right)$ ist deshalb zyklisch von der Ordnung 2; sie wird vom Bild der Derivation $D: \rho \mapsto \frac{1}{2} b_{3}+\Gamma$ erzeugt. Die Menge $\mathcal{M}_{(\Gamma, H)}$ besteht daher aus zwei Translationsklassen; sie werden durch $G_{0}=\Gamma \rtimes H$ und

$$
G_{1}=(\Gamma, \mathbb{1}) \cup(\Gamma+u, \rho) \cup\left(\Gamma+u+\rho(u), \rho^{2}\right) \cup\left(\Gamma+u+\rho(u)+\rho^{2}(u), \rho^{3}\right)
$$

vertreten. Dabei ist $u=\frac{1}{2} b_{3}=\left(\frac{1}{4}, \frac{1}{4}, \frac{1}{4}\right)^{t}$. Die Isometrie $x \mapsto \rho(x)+u$ ist eine Schraubung um die Achse $\mathbb{R} \cdot e_{3}+(0,1 / 4,0)^{t}$ mit der Ganghöhe $1 / 4$. Die Kristallographen bezeichnen die beiden Typen von Raumgruppen mit $I 4$ und $I 4_{1}$.

\subsection{Affine Typen von Raumgruppen in der Klasse $P 4 / m$}

Sei $H$ die Gruppe, die von der Drehung $\rho: x \mapsto\left(-x_{2}, x_{1}, x_{3}\right)^{t}$ und der Spiegelung $\sigma$ an der $\left(x_{1}, x_{2}\right)$-Ebene erzeugt wird; die Hermann-Mauguinsche Bezeichnung der entsprechenden geometrischen Klasse ist $\frac{4}{m}$ oder $4 / m$. Das Paar $\left(\mathbb{Z}^{3}, H\right)$ vertritt dann die arithmetische Klasse mit der Bezeichnung $P 4 / m$. Die Gruppe $H$ ist das direkte Produkt der beiden Untergruppen $S=\operatorname{gp}(\rho)$ und $T=\operatorname{gp}(\sigma)$. Da sowohl $S$ wie $T$ zyklisch sind, können wir ihre Derivationen mit Hilfssatz 6.6 bestimmen und dann mit Hilfssatz 6.7 die 
Derivationen von $H$ finden. Den Endomorphismus $\delta_{S}$ haben wir schon oben berechnet; er sendet $x$ auf $\left(0,0,4 x_{3}\right)^{t}$; der Endomorphismus $\delta_{T}=\mathbb{1}+\sigma$ hat die Beschreibung $\delta_{T}(x)=\left(2 x_{1}, 2 x_{2}, 0\right)^{t}$. Seien nun $D_{S}: S \rightarrow M$ und $D_{T}: T \rightarrow M$ Derivationen. Gemäss Hilfssatz 6.7 können sie genau dann zu einer Derivation $D: H \rightarrow M$ erweitert werden, wenn die Verträglichkeitsbedingungen

$$
D_{S}(s)+s \cdot D_{T}(t)=D_{T}(\bar{t})+\bar{t} \cdot D_{S}(\bar{s})
$$

für alle $(s, t) \in S \times T$ erfüllt sind. Da die Gruppen $S$ und $T$ miteinander kommutieren, sind $\bar{s}=s$ und $\bar{t}=t$. Weiter sind obige Bedingungen automatisch erfüllt, wenn $s$ oder $t$ das neutrale Element von $S$, beziehungsweise von $T$, ist. Es verbleiben die drei Bedingungen $D_{S}\left(\rho^{k}\right)+\rho^{k} \cdot D_{T}(\sigma)=D_{T}(\sigma)+\sigma \cdot D_{S}\left(\rho^{k}\right)$ oder

$$
(\mathbb{1}-\sigma) D_{S}\left(\rho^{k}\right)=\left(\mathbb{1}-\rho^{k}\right) D_{T}(\sigma) .
$$

Die zweite Formulierung lässt erkennen, dass die Bedingungen für $k \in\{2,3\}$ Folge der Bedingung für $k=1$, also der Bedingung $(\mathbb{1}-\sigma) D_{S}(\rho)=(\mathbb{1}-\rho) D_{T}(\sigma)$ sind.

Seien nun $R=\frac{1}{8} \mathbb{Z}, \bar{R}=R / \mathbb{Z}$ und $a, b$ zwei Elemente aus $R^{3}$ oder $\bar{R}^{3}$. Die Festsetzungen $D_{S}(\rho)=a$ und $D_{T}(\sigma)=b$ lassen sich genau dann zu einer Derivation $D$ von $H$ mit Werten in $R^{3}$, beziehungsweise in $\bar{R}^{3}$, fortsetzen, wenn die Gleichungen $\delta_{S}(a)=$ $0, \quad \delta_{T}(b)=0$ und $(\mathbb{1}-\sigma)(a)=(\mathbb{1}-\rho)(b)$ erfüllt sind; ausgeschrieben in Komponenten ergeben sie das Gleichungssystem

$$
4 a_{3}=0, \quad 2 b_{1}=2 b_{2}=0, \quad b_{1}+b_{2}=0, \quad-b_{1}+b_{2}=0, \quad 2 a_{3}=0 .
$$

Es vereinfacht sich zu $2 a_{3}=2 b_{1}=2 b_{2}=0$ und $b_{1}=b_{2}$. Die Gruppen $\operatorname{Der}\left(H, R^{3}\right)$ und $\operatorname{Der}\left(H, \bar{R}^{3}\right)$ sind deshalb isomorph mit

$$
R \oplus R \oplus\{0\} \oplus\{0\} \oplus\{0\} \oplus R \quad \text { und } \quad \bar{R} \oplus \bar{R} \oplus \frac{1}{2} \mathbb{Z} / \mathbb{Z} \oplus\left\{(\bar{r}, \bar{r}) \mid \bar{r} \in \frac{1}{2} \mathbb{Z} / \mathbb{Z}\right\} \oplus \bar{R} \text {. }
$$

Es folgt, dass die Kohomologiegruppe $H^{1}\left(H, \mathbb{R}^{3} / \mathbb{Z}^{3}\right)$ isomorph $\mathrm{zu}\left(\frac{1}{2} \mathbb{Z} / \mathbb{Z}\right)^{2}$ ist und durch die Bilder der beiden Derivationen

$$
D_{1}: \rho \mapsto\left(0,0, \frac{1}{2}\right)^{t}+\mathbb{Z}^{3}, \quad \sigma \mapsto \mathbb{Z}^{3} \quad \text { und } \quad D_{2}: \rho \mapsto \mathbb{Z}^{3}, \quad \sigma \mapsto\left(\frac{1}{2}, \frac{1}{2}, 0\right)^{t}+\mathbb{Z}^{3}
$$

erzeugt wird. Nach Satz 6.3 besteht die Menge $\mathcal{M}_{\left(\mathbb{Z}^{3}, H\right)}$ daher aus 4 Translationsklassen. Setzt man $\rho_{1}=\rho+\tau_{(0,0,1 / 2)}$ und $\sigma_{1}=\sigma+\tau_{(1 / 2,1 / 2,0)}$, so werden diese Klassen durch die 4 Gruppen $G_{(i, j)}=\operatorname{gp}\left(\mathbb{Z}^{3}, \rho_{1}^{i} \circ \sigma_{1}^{j}\right)$ vertreten, wobei $i$ und $j$ über $\{0,1\}$ variieren. Diese Gruppen sind paarweise nicht isomorph; sie vertreten daher vier eigentlich affine Typen von Raumgruppen. Die Kristallographen nennen sie $P 4 / m, P 4_{1} / m$ und $P 4 / n, P 4_{1} / n$.

\section{Ergänzungen}

Dieser Abschnitt beginnt mit einem Kommentar zur Burckhardtschen Bestimmung der 230 Typen von Raumgruppen, gefolgt von einigen Bemerkungen über eine Algorithmisierung des algebraischen Verfahrens, die auf H. Zassenhaus (1912-1991) zurückgeht ([21]; s. [14], Abschnitt 2). Der Abschnitt schliesst mit einigen Hinweisen zu neueren Arbeiten über Raumgruppen. 


\subsection{Kommentar zur Burckhardtschen Bestimmung der Raumgruppen}

Die algebraische Bestimmung der Raumgruppen eines Euklidischen Raumes $E$ setzt sich aus folgenden Teilaufgaben zusammen:

a) Erstellen einer Liste von Vertretern $(\Gamma, H)$ der arithmetischen Klassen von $E$.

b) Explizite Berechnung der Kohomologiegruppe $H^{1}(H, V / \Gamma)$ für jeden Vertreter $(\Gamma, H)$ aus der Liste.

c) Bestimmung des Normalisators $N=N_{\mathrm{GL}(\Gamma)}(H)$ und der Bahnen von $N$ auf $H^{1}(H, V / \Gamma)$ für jeden Vertreter $(\Gamma, H)$ aus der Liste.

Im Falle des 3-dimensionalen Raumes $E^{3}$ finden sich Listen von Vertretern der 73 arithmetischen Klassen bereits in den Arbeiten von Fedorov und implizit im Lehrbuch von Schoenflies. Die Teilaufgaben b) und c) musste Burckhardt hingegen als erster lösen; eine Hilfe boten ihm dabei die Resultate der geometrischen Analyse. Im Folgenden bespreche ich einige Fragen, die sich beim Lösen der Teilaufgaben b) und c) stellen.

Zu b) Die Berechnung der Kohomologiegruppe $H^{1}(H, V / \Gamma)$ führt bei 12 arithmetischen Klassen zum Ergebnis, dass jede Derivation eine innere Derivation ist. In diesen Fällen besteht die Menge $\mu_{(\Gamma, H)}$ aus einer einzigen Translationsklasse und es gibt nur einen Typ von Raumgruppen in der Klasse von $(\Gamma, H)$; er wird durch das semi-direkte Produkt $\Gamma \rtimes H$ vertreten. Für weitere 20 arithmetische Klassen ist die Kohomologiegruppe zyklisch von der Ordnung 2. Jede dieser arithmetischen Klassen enthält dann 2 Typen von Raumgruppen: ein Typ wird durch das semi-direkte Produkt vertreten, der andere Typ durch eine Gruppe, die man mittels Hilfssatz 6.1 aus einer Derivation $D: H \rightarrow V / \Gamma$, die keine innere Derivation ist, konstruiert. Bei den verbleibenden $41=73-(12+20)$ arithmetischen Klassen muss der Normalisator $N^{+}=N_{\mathrm{SL}(3, \mathbb{Z})}(H)$ herangezogen werden; siehe Nummer 7.1 und das Beispiel unten.

Da Derivationen durch ihre Werte auf einem Erzeugendensystem festgelegt werden (siehe Hilfssatz 6.5) und jede Punktgruppe $H$ von $E^{3}$ durch höchstens 3 Elemente erzeugt wird, folgt aus Satz 6.8, dass die Ordnung der Kohomologiegruppe $H^{1}(H, V / \Gamma)$ ein Teiler von $h^{3 \cdot 3}$ ist. Falls diese obere Schranke bei grösseren Punktgruppen die Ordnung der Kohomologiegruppe realistisch wiedergibt, kann die Bestimmung der Bahnen zeitraubend sein. In Wirklichkeit sind die Ordnungen der Elemente von $H^{1}(H, V / \Gamma)$ aber nur 1, 2, 3, 4 oder 6. Dieser Umstand lässt sich bei vielen arithmetischen Klassen durch das folgende Resultat erklären:

Hilfssatz 8.1 Seien $\Gamma$ ein Gitter von $E$ und $H \subseteq \mathrm{S}(\Gamma)$. Besitzt $H$ einen Normalteiler $H_{1}$, so dass kein Vektor $v$ aus $V \backslash\{0\}$ von allen Abbildungen $\psi_{1} \in H_{1}$ festgehalten wird, so ist das $\left|H_{1}\right|$-fache jeder Derivation $D: H \rightarrow V / \Gamma$ eine innere Derivation.

Beweis. Sind $\psi \in H$ und $\psi_{1} \in H_{1}$, so gilt die Rechnung

$$
D(\psi)+\bar{\psi}\left(D\left(\psi_{1}\right)\right)=D\left(\psi \cdot \psi_{1}\right)=D\left(\psi \psi_{1} \psi^{-1} \cdot \psi\right)=D\left(\psi \psi_{1} \psi^{-1}\right)+\overline{\psi \psi_{1} \psi^{-1}}(D(\psi)) .
$$

Summiert man das Resultat der vorangehenden Rechnung für alle $\psi_{1} \in H_{1}$ und berücksichtigt, dass mit $\psi_{1}$ auch $\psi \circ \psi_{1} \circ \psi^{-1}$ den Normalteiler $H_{1}$ durchläuft, so erhält man

$$
\left|H_{1}\right| \cdot D(\psi)+\bar{\psi}\left(\sum_{\psi_{1}} D\left(\psi_{1}\right)\right)=\sum_{\psi_{1}} D\left(\psi_{1}\right)+\bar{\sigma}(D(\psi))
$$


dabei bezeichnet $\sigma$ den Endomorphismus $\sum_{\psi_{1} \in H_{1}} \psi_{1}$. Dieser bildet jeden Vektor $v \in$ $V$ auf einen Vektor ab, der von allen Abbildungen $\psi_{1} \in H_{1}$ festgehalten wird; nach Annahme ist $\sigma$ also die Nullabbildung. Folglich ist $\left|H_{1}\right| \cdot D$ gleich der inneren Derivation $\psi \mapsto(\mathbb{1}-\bar{\psi})\left(\sum_{\psi_{1}} D\left(\psi_{1}\right)\right)$.

Für $V=\mathbb{R}^{3}$ lässt sich der eben bewiesene Hilfssatz insbesondere auf $H_{1}=\{\mathbb{1},-\mathbb{1}\}$ oder auf die Untergruppe, die aus $\mathbb{1}$ und den Drehungen der Ordnung 2 um die Koordinatenachsen von $\mathbb{R}^{3}$ besteht, anwenden.

Zu c) Das folgende Beispiel illustriert Hilfssatz 8.1 und zeigt auf, dass die Bestimmung der Bahnen kniffelig sein kann. Sei $H \subset \mathrm{O}(3, \mathbb{R})$ die Gruppe, die von den Spiegelungen $\sigma_{1}, \sigma_{2}$ und $\sigma_{3}$ an den Ebenen mit den Normalenvektoren $e_{1}=(1,0,0)^{t}, e_{2}$ und $e_{3}$ erzeugt wird. Die Gruppe $H$ enthält die Abbildung $-\mathbb{1}=\sigma_{3} \circ \sigma_{2} \circ \sigma_{1}$; nach Hilfssatz 8.1 teilt daher die Ordnung jedes Elementes von $H^{1}\left(H, \mathbb{R}^{3} / \mathbb{Z}^{3}\right)$ die Zahl 2; die Kohomologiegruppe ist also ein Vektorraum über dem Körper $\mathbb{F}_{2}$. Sie ist dennoch recht gross, hat sie doch 64 Elemente.

Diese Behauptung lässt sich geometrisch leicht begründen. Seien nämlich $G$ eine Raumgruppe in $\mathcal{M}_{\left(\mathbb{Z}^{3}, H\right)}$ und $\widetilde{\sigma}_{\ell}=\left(v_{\ell}, \sigma_{\ell}\right) \in G$ Urbilder der Spiegelungen $\sigma_{\ell}$. Jede der Isometrien $\widetilde{\sigma}_{\ell}$ ist die Komposition einer Spiegelung an einer zu $W_{\ell}=\left(\mathbb{R} \cdot e_{\ell}\right)^{\perp}$ parallelen Ebene und einer Translation parallel zu $W_{\ell}$. Durch Verschieben des Ursprunges kann man erreichen, dass die Spiegelungs- oder Schubspiegelungsebene die Ebene $W_{\ell}$ wird. Weil $\widetilde{\sigma}_{\ell}^{2}$ eine Translation mit einem Vektor aus $\mathbb{Z}^{3}$ sein muss, liegen die Vektoren $v_{\ell}$ zusätzlich in $\left(\frac{1}{2} \mathbb{Z}\right)^{3}$. Insgesamt genügt es daher, Isometrien der Formen

$$
\begin{aligned}
& \tilde{\sigma}_{1}=\left(\left(0, \frac{1}{2} i_{1}, \frac{1}{2} j_{1}\right)^{t}, \sigma_{1}\right), \\
& \widetilde{\sigma}_{2}=\left(\left(\frac{1}{2} i_{2}, 0, \frac{1}{2} j_{2}\right)^{t}, \sigma_{2}\right), \\
& \widetilde{\sigma}_{3}=\left(\left(\frac{1}{2} i_{3}, \frac{1}{2} j_{3}, 0\right)^{t}, \sigma_{3}\right)
\end{aligned}
$$

zu berücksichtigen; dabei durchlaufen $i_{1}, j_{1}, \ldots, i_{3}, j_{3}$ unabhängig voneinander die Menge $\{0,1\}$. Übersetzt man die gewonnene geometrische Einsicht in die Algebra, erkennt man, dass die Kohomologiegruppe $H^{1}\left(H, \mathbb{R}^{3} / \mathbb{Z}^{3}\right)$ höchstens $4^{3}=64$ Elemente hat; durch eine zusätzliche Überlegung (siehe unten) stellt man dann noch sicher, dass die Ordnung wirklich 64 ist.

Nun zu den Bahnen der Normalisatoren $N=N_{\mathrm{GL}(3, \mathbb{Z})}(H)$ und $N^{+}=N_{\mathrm{SL}(3, \mathbb{Z})}(H)$. Der Normalisator $N$ enthält die Gruppe $H$ und die 6 Permutationen der Basisvektoren $e_{1}, e_{2}$ und $e_{3}$; eine Hilfsrechnung zeigt dann, dass $N$ von diesen zwei Untergruppen erzeugt wird. Die Gruppe $H$ wirkt auf $H^{1}\left(H, \mathbb{R}^{3} / \mathbb{Z}^{3}\right)$ durch die Identität; da $H$ eine Matrix mit der Determinante -1 enthält, fallen die Bahnen der Normalisatoren $N$ und $N^{+}$auf der Kohomologiegruppe zusammen. Da jede Bahn von $N$ aus höchstens 6 Elementen besteht, gibt es mindestens $11=66 / 6$ Bahnen; eine genaue Abzählung zeigt, dass 16 Bahnen vorkommen. 1)

1) R.L.E. Schwarzenberger gibt in [18] ein graphisches Verfahren, das die Abzählung der Bahnen leicht macht; siehe auch [19], S. 88-96. 


\subsection{Algorithmus von Zassenhaus}

H. Zassenhaus publizierte 1948 einen Algorithmus zur Bestimmung der Raumgruppen eines Euklidischen Raumes E, der in zwei Punkten über das Burckhardtsche Verfahren hinausgeht:

(i) Die Bestimmung der Gruppen $\operatorname{Der}\left(H, \Gamma_{h}\right)$ und $\operatorname{Der}\left(H, \Gamma_{h} / \Gamma\right)$ läuft für jede Punktgruppe $H$, für die eine (endliche) Präsentierung durch Erzeugende und Relationen bekannt ist, auf die Berechnung eines Kernes hinaus.

(ii) (Algorithmus von Zassenhaus) Jede Präsentierung $\mathscr{P}$ von $H$ und jede $\mathbb{Z}$-Basis $\mathscr{B}$ von $\Gamma$ gibt Anlass zu einer Matrix $A=A_{\mathscr{B}, \mathscr{P}}$ mit ganzzahligen Einträgen. Durch elementare Zeilen- und Spaltenoperationen kann $A$ in eine Matrix $\hat{A}$ überführt werden, in der alle Einträge 0 sind, bis auf die Diagonaleinträge $d_{1}=\hat{A}_{11}, \ldots$, $d_{r}=\hat{A}_{r r}$. Dann ist $H^{1}(H, V / \Gamma) \approx \bigoplus_{\ell} \mathbb{Z} / d_{\ell} \mathbb{Z}$.

Im Folgenden illustriere ich beide Behauptungen durch Beispiele.

Zu Punkt (i). Sei $H \subset \mathrm{S}\left(\mathbb{Z}^{3}\right)$ die weiter oben betrachtete Gruppe der Ordnung acht, die von den Spiegelungen $\sigma_{1}, \sigma_{2}, \sigma_{3}$ an den Koordinatenebenen erzeugt wird. Seien $F$ die freie Gruppe mit den Erzeugenden $s_{1}, s_{2}$ und $s_{3}$ und $p: F \rightarrow H$ der Epimorphismus, der $s_{\ell}$ auf $\sigma_{\ell}$ sendet. Da die Spiegelungen die Ordnung 2 haben und miteinander kommutieren, enthält der Kern von $p$ die Elemente

$$
s_{1}^{2}, \quad s_{2}^{2}, \quad s_{3}^{2} \quad \text { und } \quad\left(s_{1} s_{2}\right)^{2}, \quad\left(s_{1} s_{3}\right)^{2}, \quad\left(s_{2} s_{3}\right)^{2} ;
$$

weiter ist ker $p$ gerade der von diesen Elementen erzeugte Normalteiler. Ist nun $D: H \rightarrow$ $M$ eine Derivation mit Werten in einem $H$-Modul $M$, so liefert jedes der sechs Elemente (8.1) eine Bedingung an $D$; für $\left(s_{1} s_{2}\right)^{2}=s_{1} s_{2} s_{1} s_{2}$ lautet sie

$$
\begin{aligned}
0=D(\mathbb{1})=D\left(\left(\sigma_{1} \sigma_{2}\right)^{2}\right) & =D\left(\sigma_{1}\right)+\sigma_{1} D\left(\sigma_{2}\right)+\sigma_{1} \sigma_{2} D\left(\sigma_{1}\right)+\sigma_{1} \sigma_{2} \sigma_{1} D\left(\sigma_{2}\right) \\
& =\left(\mathbb{1}+\sigma_{1} \sigma_{2}\right) D\left(\sigma_{1}\right)+\left(\mathbb{1}+\sigma_{1} \sigma_{2}\right) \sigma_{1} D\left(\sigma_{2}\right) .
\end{aligned}
$$

Setzt man $a=D\left(\sigma_{1}\right), b=D\left(\sigma_{2}\right), c=D\left(\sigma_{3}\right)$ und

$$
\tilde{A}=\left(\begin{array}{cccccc}
\mathbb{1}+\sigma_{1} & 0 & 0 & \mathbb{1}+\sigma_{1} \sigma_{2} & \mathbb{1}+\sigma_{1} \sigma_{3} & 0 \\
0 & \mathbb{1}+\sigma_{2} & 0 & \left(\mathbb{1}+\sigma_{1} \sigma_{2}\right) \sigma_{1} & 0 & \mathbb{1}+\sigma_{2} \sigma_{3} \\
0 & 0 & \mathbb{1}+\sigma_{3} & 0 & \left(\mathbb{1}+\sigma_{1} \sigma_{3}\right) \sigma_{1} & \left(\mathbb{1}+\sigma_{2} \sigma_{3}\right) \sigma_{2}
\end{array}\right),
$$

so zeigen obige Rechnung und fünf analoge Rechnungen, dass das Tripel $x=(a, b, c)$ die Matrizengleichung $x \cdot \tilde{A}=0$ erfüllt.

Da $F$ eine freie Gruppe mit der geordneten Basis $\left(s_{1}, s_{2}, s_{3}\right)$ ist, gibt es für jedes Tripel $\left(a^{\prime}, b^{\prime}, c^{\prime}\right) \in M^{3}$ eine Derivation $\tilde{D}: F \rightarrow M$ mit $\tilde{D}\left(s_{1}\right)=a^{\prime}, \tilde{D}\left(s_{2}\right)=b^{\prime}$ und $\tilde{D}\left(s_{3}\right)=c^{\prime}$. Diese Derivation $\tilde{D}$ hat genau dann die Form $\tilde{D}=D \circ p$ mit einer Derivation $D: H \rightarrow M$, wenn $\tilde{D}$ auf den Nebenklassen des Kernes von $p$ konstant ist; und letzteres tritt genau dann ein, wenn $\tilde{D}$ auf den sechs Elementen (8.1) den Wert 0 annimmt. Durch Auswerten erhält man deshalb einen Isomorphismus

$$
\operatorname{Der}(H, M) \stackrel{\sim}{\longrightarrow}\left\{(a, b, c) \in M^{3} \mid(a, b, c) \cdot \tilde{A}=0\right\} .
$$


Ersetzt man die Gruppenelemente $\sigma_{\ell}$ in $\tilde{A}$ durch die darstellenden Matrizen der Spiegelungen $\sigma_{\ell}$, erhält man eine ganzzahlige Matrix $A$ mit neun Zeilen und achtzehn Spalten. Drei ihrer Zeilen und neun ihrer Spalten bestehen nur aus Nullen. Streicht man diese Zeilen und Spalten weg, kommt man zur Matrix

$$
A_{\text {red }}=\left(\begin{array}{ccccccccc}
2 & 0 & 0 & 0 & 0 & 0 & 0 & 2 & 0 \\
0 & 2 & 0 & 0 & 0 & 0 & 2 & 0 & 0 \\
0 & 0 & 2 & 0 & 0 & 0 & 0 & 0 & 2 \\
0 & 0 & 0 & 2 & 0 & 0 & 2 & 0 & 0 \\
0 & 0 & 0 & 0 & 2 & 0 & 0 & 0 & 2 \\
0 & 0 & 0 & 0 & 0 & 2 & 0 & 2 & 0
\end{array}\right) .
$$

Ihre drei letzten Spalten sind ganzzahlige Linearkombinationen der ersten sechs Spalten und daher überflüssig. Die weggelassenen Zeilen sind die erste, die fünfte und die neunte der ursprünglichen Matrix $A$. Daher gilt

$$
\begin{aligned}
& \operatorname{Der}\left(H, R^{3}\right)=(R \oplus\{0\} \oplus\{0\}) \oplus(\{0\} \oplus R \oplus\{0\}) \oplus(\{0\} \oplus\{0\} \oplus R), \\
& \operatorname{Der}\left(H, \bar{R}^{3}\right)=\left(\bar{R} \oplus \frac{1}{2} \mathbb{Z} / \mathbb{Z} \oplus \frac{1}{2} \mathbb{Z} / \mathbb{Z}\right) \oplus\left(\frac{1}{2} \mathbb{Z} / \mathbb{Z} \oplus \bar{R} \oplus \frac{1}{2} \mathbb{Z} / \mathbb{Z}\right) \oplus\left(\frac{1}{2} \mathbb{Z} / \mathbb{Z} \oplus \frac{1}{2} \mathbb{Z} / \mathbb{Z} \oplus \bar{R}\right) .
\end{aligned}
$$

Dabei bezeichnen $R$ die Gruppe $\frac{1}{8} \mathbb{Z}$ und $\bar{R}$ die Faktorgruppe $\frac{1}{8} \mathbb{Z} / \mathbb{Z}$. Die Kohomologiegruppe $H^{1}\left(H, \mathbb{R}^{3} / \mathbb{Z}^{3}\right)$ ist somit isomorph mit $(\mathbb{Z} / 2 \mathbb{Z})^{6}$.

Zu Punkt (ii): Algorithmus von Zassenhaus. Jede Präsentierung einer Punktgruppe $H$ liefert eine Matrix $\tilde{A}$ mit Werten im Gruppenring der Gruppe $H$. Seien nun $\Gamma$ ein Gitter, das von $H$ auf sich abgebildet wird und $\mathscr{B}$ eine $\mathbb{Z}$-Basis von $\Gamma$. Ersetzt man jedes Gruppenelement $g$ in $\tilde{A}$ durch die ganzzahlige Matrix, welche die Abbildung $g: E \rightarrow$ $E$ bezüglich $\mathscr{B}$ darstellt, erhält man genau wie oben eine ganzzahlige Matrix $A$; sie hängt von den Wahlen der Präsentierung $\mathscr{P}$ und der Basis $\mathscr{P}$ ab. Bezeichnen $n$ die Dimension des Raumes, $m$ die Anzahl der Erzeugenden und $k$ die Anzahl der Relationen der Präsentierung $\mathscr{P}$, so ist $A$ eine Matrix mit $m \cdot n$ Zeilen und $k \cdot n$ Spalten. Durch elementare Zeilen- und Spaltenoperationen kann $A$ auf die in (ii) beschriebene Form $\hat{A}$ gebracht werden; anders gesagt, gibt es ganzzahlige unimodulare Matrizen $P$ und $Q$ mit $A=P \cdot \hat{A} \cdot Q$.

Die Berechnung der Gruppen $\operatorname{Der}\left(H, \Gamma_{h}\right)$ und $\operatorname{Der}\left(H, \Gamma_{h} / \Gamma\right)$ erfordert die Bestimmung der Kerne von Abbildungen

$$
\delta_{A}:\left(\frac{1}{h} \mathbb{Z}\right)^{m \cdot n} \longrightarrow\left(\frac{1}{h} \mathbb{Z}\right)^{k \cdot n}
$$

sowie

$$
\bar{\delta}_{A}:\left(\frac{1}{h} \mathbb{Z} / \mathbb{Z}\right)^{m \cdot n} \longrightarrow\left(\frac{1}{h} \mathbb{Z} / \mathbb{Z}\right)^{k \cdot n},
$$

die durch die Matrix $A$ induziert werden. Da $Q$ invertierbar ist, ändern sich diese Kerne nicht, wenn $A$ durch $P \cdot \hat{A}$ ersetzt wird. Die Matrix $P$ induziert Basiswechsel in den beiden Gruppen $\left(\frac{1}{h} \mathbb{Z}\right)^{m \cdot n}$ und $\left(\frac{1}{h} \mathbb{Z} / \mathbb{Z}\right)^{m \cdot n}$. Man kann deshalb die Kohomologiegruppe

$$
H^{1}(H, V / \Gamma) \approx \operatorname{ker}\left(\bar{\delta}_{P \cdot \hat{A}}\right) / \operatorname{im}\left(\operatorname{ker}\left(\delta_{P \cdot \hat{A}}\right) \rightarrow \operatorname{ker}\left(\bar{\delta}_{P \cdot \hat{A}}\right)\right)
$$


dadurch finden, dass man erst den Quotienten $\operatorname{ker} \bar{\delta}_{\hat{A}} / \operatorname{im}\left(\operatorname{ker} \delta_{\hat{A}} \rightarrow \operatorname{ker} \bar{\delta}_{\hat{A}}\right)$ berechnet und dann sein Bild unter der vom Basiswechsel induzierten Abbildung bestimmt. Dieser Quotient lässt sich leicht durch $\hat{A}$ beschreiben. Dazu nimmt man erst an, jedes Diagonalelement $d_{\ell}$ sei ein Teiler der Ordnung $h$. Dann sind

und

$$
\operatorname{ker} \bar{\delta}_{\hat{A}} \approx \frac{1}{d_{1}} \mathbb{Z} / \mathbb{Z} \oplus \cdots \oplus \frac{1}{d_{r}} \mathbb{Z} / \mathbb{Z} \oplus\left(\frac{1}{h} \mathbb{Z} / \mathbb{Z}\right)^{m \cdot n-r}
$$

$$
\operatorname{ker} \delta_{\hat{A}} \approx\{0\}^{r} \oplus\left(\frac{1}{h} \mathbb{Z}\right)^{m \cdot n-r}
$$

Der Quotient ist daher isomorph mit $\bigoplus_{\ell}\left(\frac{1}{d_{\ell}} \mathbb{Z} / \mathbb{Z}\right)$. Um sich von der Hilfsannahme zu befreien, geht man zum Beweis von Satz 6.8 zurück und überlegt sich, dass die Aussage des Satzes richtig bleibt, wenn die Zahl $h$ durch ein positives Vielfaches ersetzt wird. Es folgt dann wie oben, dass die Kohomologiegruppe Elemente der Ordnungen $d_{1}, \ldots, d_{r}$ besitzt. Da aber die Ordnung jedes Elementes der Kohomologiegruppe ein Teiler von $h$ ist, müssen alle Koeffizienten $d_{1}, \ldots, d_{r}$ die Ordnung $h$ der Gruppe $H$ teilen.

Beispiel. Seien $H$ die in den Anmerkungen zu Punkt (i) betrachtete Gruppe und $\Gamma=\mathbb{Z}^{3}$. Dann ist $\hat{A}$ gerade die Matrix, die von den ersten sechs Spalten der Matrix $A_{\text {red }}$ gebildet wird. Nach dem Algorithmus von Zassenhaus ist die Kohomologiegruppe $H^{1}\left(H, \mathbb{R}^{3} / \mathbb{Z}^{3}\right)$ daher isomorph $\mathrm{zu}(\mathbb{Z} / 2 \mathbb{Z})^{6}$.

\subsection{Bestimmung der arithmetischen Klassen}

Die Resultate von Zassenhaus erlauben es, die Raumgruppen eines Euklidischen Raumes E algorithmisch zu finden, sofern eine Liste von Vertretern $(\Gamma, H)$ der arithmetischen Klassen, Präsentierungen der Gruppen $H$ sowie Erzeugendensysteme der Normalisatoren $N_{\mathrm{GL}(\Gamma)}(H)$ vorliegen. Listen von Vertretern der arithmetischen Klassen kann man mit zwei Verfahren finden. Beim ersten erstellt man eine Liste von Vertretern der geometrischen Klassen und verfeinert sie danach; beim zweiten Zugang gewinnt man die Liste der Vertreter $(\Gamma, H)$ auf direktem Wege. Für beide Verfahren ist es hilfreich, über eine neue Interpretation der arithmetischen Klassen zu verfügen.

Neue Deutung der arithmetischen Klassen. Die arithmetischen Klassen sind Äquivalenzklassen von Paaren $(\Gamma, H \subseteq \mathrm{S}(\Gamma))$; man kann sie aber auch ganz anders definieren, und zwar so: Jede geordnete $\mathbb{Z}$-Basis von $\Gamma$ gibt Anlass zu einer endlichen Untergruppe $H_{\mathscr{B}}$ von $\mathrm{GL}(n, \mathbb{Z})$; ersetzt man $\mathscr{B}$ durch eine $\mathbb{Z}$-Basis $\mathscr{B}_{1}$, erhält man eine zu $H_{\mathscr{B}}$ konjugierte Untergruppe $H_{\mathscr{B}_{1}}$. Sind $\left(\Gamma^{\prime}, H^{\prime} \subseteq \mathrm{S}\left(\Gamma^{\prime}\right)\right)$ ein Paar, das arithmetisch äquivalent $\mathrm{zu}(\Gamma, H)$ ist, und $L: E^{n} \rightarrow E^{n}$ eine lineare Transformation mit $\Gamma^{\prime}=L(\Gamma)$ und $H^{\prime}=L \cdot H \cdot L^{-1}$, so ist $L(\mathscr{B})$ eine Gitterbasis von $\Gamma^{\prime}$ und die Gruppen $H_{\mathscr{乃}}$ sowie $H_{L(\mathscr{B})}^{\prime}$ fallen zusammen. Deshalb ist die Zuordnung

$$
\kappa: \mathscr{A}_{n} \longrightarrow \mathscr{C}_{n}, \quad[(\Gamma, H)] \longmapsto H_{\mathscr{B}}^{\mathrm{GL}(n, \mathbb{Z})}
$$

eine wohldefinierte Abbildung von der Menge $\mathscr{A}_{n}$ der arithmetischen Klassen von $E^{n}$ in die Menge $\mathscr{C}_{n}$ der Konjugationsklassen endlicher Untergruppen von $\operatorname{GL}(n, \mathbb{Z})$. Diese Abbildung ist bijektiv, wie man leicht nachprüft. 
Die Mengen $\mathscr{A}_{n}$ sind endlich, gilt doch der folgende Satz von Jordan [11]:

Theorem 8.2 Für jedes $n \geq 1$ besitzt $\operatorname{GL}(n, \mathbb{Z})$ nur endlich viele Konjugationsklassen endlicher Untergruppen.

Bemerkung. Unter der Korrespondenz $\kappa$ entsprechen die geometrischen Klassen von $E^{n}$ gewissen Familien von Konjugationsklassen $H^{\mathrm{GL}(n, \mathbb{Z})}$. Sie lassen sich ebenfalls arithmetisch beschreiben, und zwar als Durchschnitte $H^{\mathrm{GL}(n, \mathbb{Q})} \cap \mathrm{GL}(n, \mathbb{Z})$; siehe [12], Satz 11.5.

Bestimmung durch Verfeinerung. Die geometrischen Klassen des $E^{3}$ sind seit der Mitte des 19. Jahrhunderts bekannt. Durch Analyse der gegenseitigen Lage der Drehachsen einer kristallographischen Gruppe $H$ und eines unter $H$ invarianten Gitters kann man dann die arithmetischen Klassen finden; siehe das Beispiel in [20], Nummer 4.2. In der Kristallographie ist es dabei üblich, von einem als primitiv bezeichneten Gitter $\Gamma$ auszugehen und Obergitter zu suchen, etwa das innenzentrierte oder das fächenzentrierte. Statt dessen kann man auch versuchen, eine Übersicht über die Teilgitter $\Gamma_{1}$ eines willkürlich gewählten Gitters $\Gamma$ zu bekommen. Diese Suche nach Teilgittern lässt sich auch für Räume höherer Dimension durchführen; W. Plesken hat sie systematisiert und zu einem Algorithmus ausgebaut (siehe [14], S. 84, oder [15], Section 2).

Endliche Untergruppen von GL $(n, \mathbb{Z})$ können andererseits mit Hilfe der Darstellungstheorie gefunden werden. Auf diesem Wege konstruierte bereits Frobenius 1911 Vertreter der 32 geometrischen Kristallklassen des $E^{3}$ (siehe [10]).

Direkte Bestimmung. Die Zahl der arithmetischen Klassen des $E^{3}$ ist 73 , also schon recht gross. Wie in [1] vorgeführt wird, kann man Vertreter aller Klassen aber immer noch durch direkte algebraische Rechnungen finden.

Dieses Ziel kann man auf einem anderen Wege erreichen, der auch in höheren Dimensionen gangbar ist. Dazu nützt man aus, dass jede endliche Untergruppe $H$ von $\operatorname{GL}(n, \mathbb{Z})$ in einer maximal endlichen Untergruppe $\tilde{H}$ enthalten ist (dies ergibt sich etwa aus der Bemerkung 2) in [20], Nummer 2.3). Die Zahl der Konjugationsklassen maximal endlicher Untergruppen für $n \leq 3$ ist vergleichsweise klein: für $n=2$ gibt es zwei, für $n=3$ nur vier derartige Konjugationsklassen; sie entsprechen den Symmetriegruppen des quadratischen und des hexagonalen ebenen Gitters, beziehungsweise jenen des primitiven, des innenzentrierten und des flächenzentrierten kubischen Gitters sowie des hexagonalen Gitters von $E^{3}$. Wenn es gelingt, diese wenigen Gruppen $\tilde{H}$ direkt zu bestimmen, genügt es, in einem zweiten Schritt die Untergruppen der maximal endlichen Gruppen $\tilde{H}$ aufzulisten und bis auf Konjugation zu klassifizieren.

E.C. Dade gelang es 1965 , die maximal endlichen Untergruppen von $\mathrm{GL}(4, \mathbb{Z})$ direkt zu finden: es gibt neun Konjugationsklassen solcher Gruppen; ihre grösste hat die Ordnung $1152=2^{7} \cdot 3^{2}$ (siehe [8]). Gestützt auf diese Liste konnten schon 1967 die 710 arithmetischen Klassen des $E^{4}$ mit Hilfe eines Grossrechners bestimmt werden ([2], S. 1-4, gibt weitere Details). Die maximal endlichen Untergruppen von $\operatorname{GL}(n, \mathbb{Z})$ sind später auch für Dimensionen $n>4$ bestimmt worden, so um 1972 für $n=5$ durch R. Bülow und durch S.S. Ryskov (genauere Angaben finden sich im Übersichtsartikel [14] von W. Plesken). 


\section{Literatur}

[1] Auslander, L.; Cook, M.: An algebraic classification of the three-dimensional crystallographic groups. Adv. Appl. Math. 12 (1991), 1-21.

[2] Brown, H.; Bülow, R.; Neubüser, J.; Wondratschek, H.; Zassenhaus, H.: Crystallographic groups of four-dimensional space. John Wiley \& Sons, 1978.

[3] Burckhardt, J.J.: Bemerkungen zur arithmetischen Berechnung der Bewegungsgruppen. Comment. Math. Helv. 2 (1930), 91-98.

[4] Burckhardt, J.J.: Zur Theorie der Bewegungsgruppen. Comment. Math. Helv. 6 (1934), 159-184.

[5] Burckhardt, J.J.: Bewegungsgruppen in mehrdimensionalen Räumen. Comment. Math. Helv. 9 (1936/37), 284-302.

[6] Burckhardt, J.J.: Die Bewegungsgruppen der Kristallographie. Birkhäuser, 1947 (erste Auflage) und 1966 (zweite, neubearbeitete Auflage).

[7] Conway, J.H.; Delgado Friedrichs, O.; Huson, D.H.; Thurston, W.P.: On Three-Dimensional Space Groups. Beitr. Alg. Geometrie 42 (2001), 475-507.

[8] Dade, E.C.: The maximal finite groups of $4 \times 4$ integral matrices. Illinois J. Math. 9 (1965), 99-122.

[9] Frobenius, E.S.: Über die unzerlegbaren diskreten Bewegungsgruppen. Sitzungsber. K. Preuss. Akad. Wiss. Berlin (1911), 654-665; Ges. Abh. Bd. 3, 507-518.

[10] Frobenius, E.S.: Gruppentheoretische Ableitung der 32 Kristallklassen. Sitzungsber. K. Preuss. Akad. Wiss. Berlin (1911), 681-691; Ges. Abh. Bd. 3, 519-529.

[11] Jordan, C.: Mémoire sur l'équivalence des formes. J. Éc. Pol. 48 (1880), 112-150; CEuvres, Vol. III, $421-460$.

[12] Klemm, M.: Symmetrien von Ornamenten und Kristallen. Springer, 1982.

[13] Maxwell, G.: The crystallography of Coxeter groups. J. Alg. 35 (1975), 159-177.

[14] Plesken, W.: Kristallographische Gruppen. In: Group Theory, Algebra, Number Theory (ed. Zimmer, H.G.), Walter de Gruyter \& Co., 1996, 75-96.

[15] Plesken, W.; Pohst, M.: On maximal finite irreducible subgroups of $\operatorname{GL}(n, \mathbb{Z})$. I. The five and seven dimensional cases. Math. Comp. 31 (1977), 536-551.

[16] Plesken, W.; Schulz, T.: Counting crystallographic groups in low dimensions. Exp. Math. 9 (2000), 407-411.

[17] Schoenflies, A.: Krystallsysteme und Krystallstructur. Teubner, 1891.

[18] Schwarzenberger, R.L.E.: The use of directed graphs in the enumeration of orthogonal space groups. Acta Crystallographica A32 (1976), 356-359.

[19] Schwarzenberger, R.L.E.: N-dimensional crystallography. Pitman Advanced Publishing Program, 1980.

[20] Strebel, R.: Burckhardtsche Bestimmung der Raumgruppen I. Elem. Math. 58 (2003), 141-155.

[21] Zassenhaus, H.: Über einen Algorithmus zur Bestimmung der Raumgruppen. Comment. Math. Helv. 21 (1948), 117-141.

Ralph Strebel

Departement für Mathematik

Universität Freiburg

CH-1700 Freiburg, Schweiz

e-mail: ralph.strebel@unifr.ch 